\title{
BOUNDS ON ENERGY AND LAPLACIAN ENERGY OF GRAPHS
}

\author{
SRIDHARA G ${ }^{1,2}$, M.R.RAJESH KANNA 3,4 \\ ${ }^{1,3}$ Post Graduate Department of Mathematics, \\ Maharani's Science College for Women, \\ J. L. B. Road, Mysore - 570 005, India. \\ ${ }^{2}$ Research Scholar, Research and Development Centre, \\ Bharathiar University, Coimbatore 641 046, India \\ srsrig@gmail.com \\ ${ }^{4}$ Government First Grade College, Bettampady, Puttur,D.K, India \\ mr.rajeshkanna@gmail.com
}

\begin{abstract}
Let $G$ be simple graph with $n$ vertices and $m$ edges. The energy $E(G)$ of $G$, denoted by $E(G)$, is defined to be the sum of the absolute values of the eigenvalues of $G$. In this paper, we present two new upper bounds for energy of a graph, one in terms of $m, n$ and another in terms of largest absolute eigenvalue and the smallest absolute eigenvalue. The paper also contains upper bounds for Laplacian energy of graph.
\end{abstract}

Key words and Phrases: Adjacency matrix, Laplacian matrix, Energy of graph, Laplacian energy of graph.

\begin{abstract}
Abstrak. Misalkan $G$ adalah graf sederhana dengan $n$ titik dan $m$ sisi. Energi $E(G)$ dari $G$, dinotasikan dengan $E(G)$, didefinisikan sebagai jumlahan dari nilai mutlak dari nilai-nilai eigen $G$. Pada paper ini, kami menyatakan dua batas atas baru untuk energi dari graf, satu batas dalam suku $m, n$ dan batas yang lain dalam suku nilai eigen mutlak terbesar dan terkecil. Paper ini juga memuat batas atas untuk energi Laplace dari graf.
\end{abstract}

Kata kunci: Matriks ketetanggaan, matriks Laplace, energi dari graf, energi Laplace dari graf.

2000 Mathematics Subject Classification: Primary 05C50, 05C69.

Received: 26 Sept 2016, revised: 25 March 2017, accepted: 26 March 2017. 


\section{INTRODUCTION}

The concept of energy of a graph was introduced by I. Gutman [6] in the year 1978. Let $G$ be a graph with $n$ vertices $\left\{v_{1}, v_{2}, \ldots, v_{n}\right\}$ and $m$ edges and $A=\left(a_{i j}\right)$ be the adjacency matrix of the graph. The eigenvalues $\lambda_{1}, \lambda_{2}, \cdots, \lambda_{n}$ of $\mathrm{A}$, assumed in non increasing order, are the eigenvalues of the graph $G$. The energy $E(G)$ of $G$ is defined to be the sum of the absolute values of the eigenvalues of $G$. i.e., $E(G)=$ $\sum_{i=1}^{n}\left|\lambda_{i}\right|$. For details on the mathematical aspects of the theory of graph energy see the papers $[2,3,8]$ and the references cited there in. The basic properties including various upper and lower bounds for energy of a graph have been established in [10] and it has found remarkable chemical applications in the molecular orbital theory of conjugated molecules $[5,9]$. The bounds for eigenvalues of graph can be found in $[1,13]$.

Definition 1.1. Let $G$ be a graph with $n$ vertices and $m$ edges. The Laplacian matrix of the graph $G$, denoted by $L=\left(L_{i j}\right)$, is a square matrix of order $n$ whose elements are defined as

$$
L_{i j}= \begin{cases}-1 & \text { if } v_{i} \text { and } v_{j} \text { are adjacent } \\ 0 & \text { if } v_{i} \text { and } v_{j} \text { are not adjacent } \\ d_{i} & \text { if } i=j\end{cases}
$$

where $d_{i}$ is the degree of the vertex $v_{i}$.

Eigenvalues of $L$ is called eigenvalues of $G$.

Definition 1.2. Let $\mu_{1}, \mu_{2}, \cdots, \mu_{n}$ be the Laplacian eigenvalues of G. Laplacian energy $L E(G)$ of $G$ is defined as $L E(G)=\sum_{i=1}^{n}\left|\mu_{i}-\frac{2 m}{n}\right|$.

The matrix $L$ is positive semi-definite and therefore its eigenvalues are nonnegative. The least eigenvalue is always equal to zero. The second largest eigenvalue is called the algebraic connectivity of $G$. The basic properties including various upper and lower bounds for Laplacian energy have been established in $[7,11,12,13]$.

\section{Main Results}

2.1. Energy of graph. We denote the decreasing order of the the absolute value of eigenvalues of $G$ by $\rho_{1} \geq \rho_{2} \geq \ldots \geq \rho_{n}$. The following are the elementary results that follows from this notation.
(1) $\rho_{i}=\left|\lambda_{k}\right|$ for some $k$
(2) $\rho_{i} \geq \lambda_{i}$ for all i
(3) $E(G)=\sum_{i=1}^{n} \rho_{i}$ 
(4) $\rho_{n} \leq \sum_{i=1}^{n} \rho_{i}=E(G)$

(5) By Cauchy-Schwarz inequality

$$
\begin{aligned}
& \left(\sum_{i=1}^{n} \lambda_{i} \rho_{i}\right)^{2} \leq\left(\sum_{i=1}^{n} \rho_{i}^{2}\right)\left(\sum_{i=1}^{n} \lambda_{i}^{2}\right) \\
& \sum_{i=1}^{n} \lambda_{i} \rho_{i} \leq \sqrt{(2 m)(2 m)}
\end{aligned}
$$

Therefore $\sum_{i=1}^{n} \lambda_{i} \rho_{i} \leq 2 m$, equality holds if $\rho_{i}=\lambda_{i}$.

(6) Let $G$ and $H$ be any two graphs with same $n$ vertices each. Let their number of edges be respectively $m_{1}$ and $m_{2}$. If $\rho_{1} \geq \rho_{2} \geq \ldots \geq \rho_{n}$ and $\rho_{1}^{\prime} \geq \rho_{2}^{\prime} \geq \ldots \geq \rho_{n}^{\prime}$ are their the absolute value of eigenvalues then

$$
\begin{aligned}
\sum_{i=1}^{n} \rho_{i} \rho_{i}^{\prime} & \leq \sqrt{\left(\sum_{i=1}^{n} \rho_{i}^{2}\right)\left(\sum_{i=1}^{n} \rho_{i}^{2}\right)} \\
& \leq \sqrt{\left(2 m_{1}\right)\left(2 m_{2}\right)} \\
\therefore \quad \sum_{i=1}^{n} \rho_{i} \rho_{i}^{\prime} & \leq 2 \sqrt{m_{1} m_{2}}
\end{aligned}
$$

(7) Since $\lambda_{1}$ is always positive, so $\rho_{1}=\lambda_{1} \geq \frac{2 m}{n}$

(8) Since $n \rho_{n}^{2} \leq \rho_{1}^{2}+\rho_{2}^{2}+\ldots+\rho_{n}^{2}=2 m$ which implies $\rho_{n} \leq \sqrt{\frac{2 m}{n}}$

Theorem 2.1. Let $G$ be a graph with $n$ vertices and $m$ edges. Let $\rho_{1} \geq \rho_{2} \geq \ldots \geq$ $\rho_{n}$ be the the absolute value of eigenvalues of $G$ then $\rho_{n} \leq \sqrt{\frac{2 m(n-1)}{n}}$.

Proof. We know that $E(G)=\sum_{i=1}^{n} \rho_{i}$ and $\sum_{i=1}^{n} \rho_{i}^{2}=2 m$

Since $\rho_{n} \leq \rho_{i} \forall i \quad \therefore \quad \rho_{n} \leq \sum_{i=1}^{n-1} \rho_{i}$

By Cauchy Schwarz inequality $\left(\sum_{i=1}^{n-1} \rho_{i}\right)^{2} \leq \sum_{i=1}^{n-1} 1^{2} \sum_{i=1}^{n-1} \rho_{i}^{2}$

$$
\begin{array}{r}
=(n-1) \sum_{i=1}^{n-1} \rho_{i}^{2} \\
\Rightarrow \sum_{i=1}^{n-1} \rho_{i}^{2} \geq \frac{1}{(n-1)}\left(\sum_{i=1}^{n-1} \rho_{i}\right)^{2}
\end{array}
$$




$$
\begin{aligned}
2 m-\rho_{n}^{2} & \geq \frac{1}{(n-1)}\left(\sum_{i=1}^{n-1} \rho_{i}\right)^{2} \\
& \geq \frac{1}{(n-1)} \rho_{n}^{2} \\
\Rightarrow \rho_{n} & \leq \sqrt{\frac{2 m(n-1)}{n}}
\end{aligned}
$$

which is an upper bound for the smallest absolute eigenvalue of the graph $G$

Theorem 2.2. Let $G$ be a graph with $n$ vertices and $m$ edges. Let $\rho_{1} \geq \rho_{2} \geq \ldots \geq$ $\rho_{n}$ be the the absolute value of eigenvalues of $G$. If $\rho_{1}$ is repeated $k$ times then

$$
\rho_{1} \leq \frac{1}{k(p-1)}\left(\sqrt{2 m k p(p-1)}-\sum_{i=k+1}^{k p} \rho_{i}\right) \text { where } k p \leq n \text { and } p \neq 1, k \neq 0 .
$$

Proof. Let $H=\left(\bigcup_{k} K_{p}\right) \cup\left(K_{n-k p}\right)^{c}$ where $k p \leq n$

That is $H$ is the union of graphs $K_{p}$, repeated $k$ times and a graph $\left(K_{n-k p}\right)^{c}$.

The number of vertices of $H$ is $n$ and the number of edges is $\frac{k p(p-1)}{2}$. Its the absolute value of eigenvalues spectrum is

$$
\left(\begin{array}{ccc}
p-1 & 1 & 0 \\
k & k(p-1) & (n-k p)
\end{array}\right) .
$$

By Cauchy Schwarz inequality

$$
\begin{aligned}
& \rho_{1}(p-1)+\ldots+\rho_{k}(p-1)+\rho_{k+1}(1)+\ldots+\rho_{k p}(1)+\rho_{k p+1}(0)+\ldots+\rho_{n}(0) \leq 2 \sqrt{m \frac{k p(p-1)}{2}} \\
& \text { But } \rho_{1}=\rho_{2}=\ldots=\rho_{k} \\
& \therefore(p-1) k \rho_{1}+\sum_{i=k+1}^{k p} \rho_{i} \leq 2 \sqrt{m \frac{k p(p-1)}{2}} \\
& \rho_{1} \leq \frac{1}{k(p-1)}\left(\sqrt{2 m k p(p-1)}-\sum_{i=k+1}^{k p} \rho_{i}\right) . \text { Here }(p \neq 1, k \neq 0)
\end{aligned}
$$

Corollary 2.3. If $k p=n$, then by the above theorem

$$
\begin{aligned}
& (n-k) \rho_{1}+\sum_{i=k+1}^{n} \rho_{i} \leq \sqrt{\frac{2 m n(n-k)}{k}} \\
& (n-k) \rho_{1}+E(G)-k \rho_{1} \leq \sqrt{\frac{2 m n(n-k)}{k}}
\end{aligned}
$$




$$
\begin{aligned}
& (n-2 k) \rho_{1}+E(G) \leq \sqrt{\frac{2 m n(n-k)}{k}} \\
& E(G) \leq \sqrt{\frac{2 m n(n-k)}{k}}-(n-2 k) \rho_{1}
\end{aligned}
$$

Also if $p=2$ and $2 k=n$ then the upper bound for energy of graph is

$E(G) \leq \sqrt{\frac{2 m n(2 k-k)}{k}}$

$E(G) \leq \sqrt{2 m n}$.

Corollary 2.4. If $k p=n-1$, then we get the following result.

$$
\begin{aligned}
& E(G)-\rho_{n} \leq \sqrt{\frac{2 m(n-1)(n-1-k)}{k}}-(n-1-2 k) \rho_{1} \\
& E(G) \leq \sqrt{\frac{2 m(n-1)(n-1-k)}{k}}-(n-1-2 k) \rho_{1}+\rho_{n} .
\end{aligned}
$$

Also if $p=2$ and $2 k=n-1$ then the upper bound for energy of graph is $E(G) \leq \sqrt{2 m(n-1)}+\rho_{n}$.

Corollary 2.5. If $k=1$, then $E(G) \leq \sqrt{2 m n(n-1)}-(n-2) \rho_{1} \quad$ for $p=n$. and $E(G) \leq \sqrt{2 m(n-1)(n-2)}-(n-3) \rho_{1}+\rho_{n}$ for $p=n-1$.

Corollary 2.6. Since $\rho_{1} \geq \frac{2 m}{n}$ and $\rho_{n} \leq \sqrt{\frac{2 m}{n}}$ we get new upper bound for energy of graph in term of $m$ and $n$

$$
\begin{aligned}
& E(G) \leq \sqrt{\frac{2 m n(n-k)}{k}}-(n-2 k) \frac{2 m}{n} \text { for } p k=n . \\
& E(G) \leq \sqrt{\frac{2 m(n-1)(n-1-k)}{k}}-(n-1-2 k) \frac{2 m}{n}+\sqrt{\frac{2 m}{n}} \text { for } p k=n-1 .
\end{aligned}
$$

Corollary 2.7. For a r-regular graph $m=\frac{r n}{2}$ and $\rho_{1}=r$ we have the following upper bound

$$
\begin{aligned}
& E(G) \leq n \sqrt{\frac{r(n-k)}{k}}-(n-2 k) r \text { for } p k=n . \\
& E(G) \leq \sqrt{\frac{r n(n-1)(n-1-k)}{k}}-(n-1-2 k) r+\sqrt{r} \text { for } p k=n-1 .
\end{aligned}
$$


Theorem 2.8. Let $G$ be a graph with $n$ vertices and $m$ edges. Let $\rho_{1} \geq \rho_{2} \geq \ldots \geq$ $\rho_{n}$ be the the absolute value of eigenvalues of $G$. If $\rho_{1}$ is repeated $k$ times then $\rho_{1} \leq \frac{1}{k}\left(2 \sqrt{m k}-\sum_{i=k+1}^{2 k} \rho_{i}\right) .(k \neq 0)$

Proof. Here we compare the absolute value of eigenvalues of $G$ with absolute eigenvalue of the graph $H=\left(\bigcup_{k} K_{p, q}\right)$.

Select $p$ and $q$ such that $n=k(p+q)$. The number of vertices of $H$ is $n$ and the number of edges is $k p q$. Its the absolute value of eigenvalues spectrum are

$$
\left(\begin{array}{cc}
\sqrt{p q} & 0 \\
2 k & (n-2 k)
\end{array}\right) .
$$

By Cauchy Schwarz inequality

$\rho_{1} \sqrt{p q}+\ldots+\rho_{k} \sqrt{p q}+\rho_{k+1} \sqrt{p q}+\ldots+\rho_{2 k} \sqrt{p q}+\rho_{2 k+1}(0)+\ldots+\rho_{n}(0) \leq 2 \sqrt{m k p q}$

But $\rho_{1}=\rho_{2}=\ldots=\rho_{k}$

$$
\begin{aligned}
& \therefore \quad \rho_{1} k \sqrt{p q}+\sqrt{p q} \sum_{i=k+1}^{2 k} \rho_{i} \leq 2 \sqrt{m k p q} \\
& \rho_{1} k+\sum_{i=k+1}^{2 k} \rho_{i} \leq 2 \sqrt{m k} \\
& \rho_{1} \leq \frac{1}{k}\left(2 \sqrt{m k}-\sum_{i=k+1}^{2 k} \rho_{i}\right) .
\end{aligned}
$$

Corollary 2.9. If $p=q=1$ and $2 k=n$ then

$$
\begin{aligned}
& \rho_{1} k+\sum_{i=k+1}^{n} \rho_{i} \leq 2 \sqrt{m \frac{n}{2}} \\
& \text { i.e., } E(G) \leq \sqrt{2 m n} .
\end{aligned}
$$

Corollary 2.10. If $p=q=1$ and $2 k=n-1$ then

$$
\begin{aligned}
& \rho_{1} k+\sum_{i=k+1}^{n-1} \rho_{i} \leq 2 \sqrt{m \frac{(n-1)}{2}} \\
& \Rightarrow E(G)-\rho_{n} \leq \sqrt{2 m(n-1)} \\
& \text { i.e., } E(G) \leq \sqrt{2 m(n-1)}+\rho_{n}
\end{aligned}
$$




$$
\text { i.e., } E(G) \leq \sqrt{2 m(n-1)}+\sqrt{\frac{2 m}{n}} .
$$

Corollary 2.11. For $k=1, \rho_{1}+\rho_{2} \leq 2 \sqrt{m}$.

Using the above corollary we obtain another bound for energy of graphs.

Theorem 2.12. Let $G$ be a graph with $n$ vertices and $m$ edges and $2 m \geq n$. If the first absolute eigenvalue, $\rho_{1}$ not repeated then $E(G) \leq \sqrt{m}(2+\sqrt{2 n-4})$

Proof. Cauchy Schwarz inequality for $(n-2)$ terms is

$$
\left(\sum_{i=3}^{n} a_{i} b_{i}\right)^{2} \leq\left(\sum_{i=3}^{n} a_{i}^{2}\right)\left(\sum_{i=3}^{n} b_{i}^{2}\right)
$$

Put $a_{i}=\rho_{i}$ and $b_{i}=1$

$$
\begin{aligned}
& \sum_{i=3}^{n} \rho_{i} \leq \sqrt{\left(\sum_{i=3}^{n} \rho_{i}^{2}\right)\left(\sum_{i=3}^{n} 1\right)} \\
& E(G)-\left(\rho_{1}+\rho_{2}\right) \leq \sqrt{\left(2 m-\left(\rho_{1}^{2}+\rho_{2}^{2}\right)\right)(n-2)} \\
& E(G) \leq\left(\rho_{1}+\rho_{2}\right)+\sqrt{n-2} \sqrt{\left(2 m-\left(\rho_{1}^{2}+\rho_{2}^{2}\right)\right)}
\end{aligned}
$$

But $\rho_{1}+\rho_{2} \leq 2 \sqrt{m} \quad \therefore \quad E(G) \leq 2 \sqrt{m}+\sqrt{n-2} \sqrt{\left(2 m-\left(\rho_{1}^{2}+\rho_{2}^{2}\right)\right)}$

We maximize the function $f(x, y)=2 \sqrt{m}+\sqrt{n-2} \sqrt{\left(2 m-\left(x^{2}+y^{2}\right)\right)}$

Then $f_{x}=\frac{-\sqrt{n-2} x}{\sqrt{\left(2 m-\left(x^{2}+y^{2}\right)\right)}}$ and $f_{y}=\frac{-\sqrt{n-2} y}{\sqrt{\left(2 m-\left(x^{2}+y^{2}\right)\right)}}$

For maxima value $f_{x}=0$ and $f_{y}=0$ which implies $(x, y) \equiv(0,0)$

$$
\begin{gathered}
f_{x x}=\frac{-\sqrt{n-2}\left(2 m-y^{2}\right)}{\left(2 m-\left(x^{2}+y^{2}\right)\right)^{\frac{3}{2}}}, f_{y y}=\frac{-\sqrt{n-2}\left(2 m-x^{2}\right)}{\left(2 m-\left(x^{2}+y^{2}\right)\right)^{\frac{3}{2}}}, f_{x y}=\frac{\sqrt{n-2} x y}{\left(2 m-\left(x^{2}+y^{2}\right)\right)^{\frac{3}{2}}} \\
\text { At }(x, y) \equiv(0,0), f_{x x}=-\sqrt{\frac{n-2}{2 m}}, f_{y y}=-\sqrt{\frac{n-2}{2 m}}, f_{x y}=0 \text { and } \\
\Delta=f_{x x} f_{y y}-\left(f_{x y}\right)^{2}=\frac{n-2}{2 m}
\end{gathered}
$$

Thus $f(x, y)$ attains maximum value at $(0,0) \therefore \quad f(0,0)=\sqrt{m}(2+\sqrt{2 n-4})$

$$
E(G) \leq \sqrt{m}(2+\sqrt{2 n-4}) .
$$

2.2. Laplacian energy of graph. Analogous to the bounds for energy of graphs, now we obtain bounds for Laplacian energy of graphs.

Theorem 2.13. Let $G$ and $H$ are two graphs with $n$ vertices each. Let their number of edges be respectively be $m_{1}$ and $m_{2}$. If $\sigma_{1} \geq \sigma_{2} \geq \ldots \geq \sigma_{n}$ represent 
absolute Laplacian eigenvalues of $G$ and $\lambda_{1} \geq \lambda_{2} \geq \ldots \geq \lambda_{n}$ eigenvalues of $H$ then $\sum_{i=1}^{n} \sigma_{i} \lambda_{i} \leq \sqrt{\left(2 m_{2}\right)\left(2 m_{1}+\sum_{i=1}^{n}\left(d_{i}(G)\right)^{2}\right)}$ where $d_{i}(G)$ is the degree of the vertex $v_{i}$.

Proof. By Cauchy Schwarz inequality

$$
\begin{gathered}
\sum_{i=1}^{n} \sigma_{i} \lambda_{i} \leq \sqrt{\left(\sum_{i=1}^{n} \sigma_{i}^{2}\right)\left(\sum_{i=1}^{n} \lambda_{i}^{2}\right)} \\
\text { But } \quad \sum_{i=1}^{n} \sigma_{i}^{2}=\left(2 m_{1}+\sum_{i=1}^{n}\left(d_{i}(G)\right)^{2}\right) \\
\therefore \quad \sum_{i=1}^{n} \sigma_{i} \lambda_{i} \leq \sqrt{\left(2 m_{2}\right)\left(2 m_{1}+\sum_{i=1}^{n}\left(d_{i}(G)\right)^{2}\right)} .
\end{gathered}
$$

Theorem 2.14. Let $G$ be a graph with $n$ vertices and $m$ edges. Let $\sigma_{1} \geq \sigma_{2} \geq$ $\ldots \geq \sigma_{n}$ be the absolute Laplacian eigenvalues of $G$. If $\sigma_{1}$ is repeated $k$ times then

$$
\sigma_{1} \leq \frac{1}{k(p-1)}\left(\sqrt{\left(2 m+\sum_{i=1}^{n}\left(d_{i}(G)\right)^{2}\right) \frac{k p(p-1)}{2}}-\sum_{i=k+1}^{k p} \sigma_{i}\right)
$$

where $k p \leq n, \quad k \neq 0, p \neq 1$

Proof. Let $H=\left(\bigcup_{k} K_{p}\right) \cup\left(K_{n-k p}\right)^{c}$ where $k p \leq n$

That is $H$ is union of graphs $K_{p}$, repeated $k$ times and a graph $\left(K_{n-k p}\right)^{c}$.

The number of vertices of $H$ is $n$ and the number of edges is $\frac{k p(p-1)}{2}$. Its the absolute value of eigenvalues spectrum is

$$
\left(\begin{array}{ccc}
p-1 & 1 & 0 \\
k & k(p-1) & (n-k p)
\end{array}\right) .
$$

By Cauchy Schwarz inequality

$$
\begin{aligned}
& \sigma_{1}(p-1)+\sigma_{2}(p-1)+\ldots+\sigma_{k}(p-1)+\sigma_{k+1}(1)+\sigma_{k+2}(1)+\ldots+\sigma_{k p}(1)+\sigma_{k p+1}(0)+ \\
& \ldots+\sigma_{n}(0) \leq \sqrt{\left(2 m+\sum_{i=1}^{n}\left(d_{i}(G)\right)^{2}\right) \frac{k p(p-1)}{2}}
\end{aligned}
$$

But $\sigma_{1}=\sigma_{2}=\ldots=\sigma_{k}$

$$
(p-1) k \sigma_{1}+\sum_{i=k+1}^{k p} \sigma_{i} \leq \sqrt{\left(2 m+\sum_{i=1}^{n}\left(d_{i}(G)\right)^{2}\right) \frac{k p(p-1)}{2}}
$$




$$
\begin{aligned}
& k \sigma_{1} \leq \frac{1}{p-1}\left(\sqrt{\left(2 m+\sum_{i=1}^{n}\left(d_{i}(G)\right)^{2}\right) \frac{k p(p-1)}{2}}-\sum_{i=k+1}^{k p} \sigma_{i}\right) \\
& \sigma_{1} \leq \frac{1}{k(p-1)}\left(\sqrt{\left(2 m+\sum_{i=1}^{n}\left(d_{i}(G)\right)^{2}\right) \frac{k p(p-1)}{2}}-\sum_{i=k+1}^{k p} \sigma_{i}\right) .
\end{aligned}
$$

Corollary 2.15. If $k p=n$ then by the above theorem

$$
\begin{aligned}
& (n-k) \sigma_{1}+\sum_{i=k+1}^{k p} \sigma_{i} \leq \sqrt{\left(2 m+\sum_{i=1}^{n}\left(d_{i}(G)\right)^{2}\right) \frac{n(n-k)}{k}} \\
& (n-k) \sigma_{1}+L E(G)-k \sigma_{1} \leq \sqrt{\left(2 m+\sum_{i=1}^{n}\left(d_{i}(G)\right)^{2}\right) \frac{n(n-k)}{k}} \\
& (n-2 k) \sigma_{1}+L E(G) \leq \sqrt{\left(2 m+\sum_{i=1}^{n}\left(d_{i}(G)\right)^{2}\right) \frac{n(n-k)}{k}} \\
& L E(G) \leq \sqrt{\left(2 m+\sum_{i=1}^{n}\left(d_{i}(G)\right)^{2}\right) \frac{n(n-k)}{k}}-(n-2 k) \sigma_{1} .
\end{aligned}
$$

Also if $p=2$ and $2 k=n$ then the upper bound for Laplacian energy of graph is

$$
\begin{aligned}
& L E(G) \leq \sqrt{\left(2 m+\sum_{i=1}^{n}\left(d_{i}(G)\right)^{2}\right) \frac{n(2 k-k)}{k}} \\
& L E(G) \leq \sqrt{\left(2 m+\sum_{i=1}^{n}\left(d_{i}(G)\right)^{2}\right) n .}
\end{aligned}
$$

Corollary 2.16. If $k p=n-1$ we get the following result.

$$
\begin{aligned}
& L E(G)-\sigma_{n} \leq \sqrt{\left(2 m+\sum_{i=1}^{n}\left(d_{i}(G)\right)^{2}\right) \frac{(n-1)(n-1-k)}{k}}-(n-1-2 k) \sigma_{1} \\
& L E(G) \leq \sqrt{\left(2 m+\sum_{i=1}^{n}\left(d_{i}(G)\right)^{2}\right) \frac{(n-1)(n-1-k)}{k}}-(n-1-2 k) \sigma_{1}+\sigma_{n}
\end{aligned}
$$

Also if $p=2$ and $2 k=n-1$ then we get the following upper bound for Laplacian energy of graph

$$
\begin{aligned}
& L E(G) \leq \sqrt{\left(2 m+\sum_{i=1}^{n}\left(d_{i}(G)\right)^{2}\right) \frac{(n-1)(2 k-k)}{k}}+\sigma_{n} \\
& L E(G) \leq \sqrt{\left(2 m+\sum_{i=1}^{n}\left(d_{i}(G)\right)^{2}\right)(n-1)}+\sigma_{n} .
\end{aligned}
$$

Corollary 2.17. If $k=1$ then the upper bounds changes to $L E(G) \leq \sqrt{\left(2 m+\sum_{i=1}^{n}\left(d_{i}(G)\right)^{2}\right) n(n-1)}-(n-2) \sigma_{1}$ for $p=n$ 
$L E(G) \leq \sqrt{\left(2 m+\sum_{i=1}^{n}\left(d_{i}(G)\right)^{2}\right)(n-1)(n-2)}-(n-3) \sigma_{1}+\sigma_{n}$ for $p=n-1$.

Theorem 2.18. Let $G$ be a graph with $n$ vertices and $m$ edges. Let $\sigma_{1} \geq \sigma_{2} \geq$ $\ldots \geq \sigma_{n}$ be the absolute Laplacian eigenvalues of $G$. If $\sigma_{1}$ is repeated $k$ times then $\sigma_{1} \leq \frac{1}{k}\left(\sqrt{\left(2 m+\sum_{i=1}^{n}\left(d_{i}(G)\right)^{2}\right) 2 k}-\sum_{i=k=1}^{2 k} \sigma_{i}\right) \quad(k \neq 0)$.

Proof. Here we compare absolute Laplacian eigenvalues of $G$ with absolute eigenvalue of graph $H=\left(\bigcup_{k} K_{p, q}\right)$.

Select $p$ and $q$ such that $n=k(p+q)$. The number of vertices of $H$ is $n$ and the number of edges is $k p q$. Its the absolute value of eigenvalues spectrum is $\left(\begin{array}{cc}\sqrt{p q} & 0 \\ 2 k & (n-2 k)\end{array}\right)$.

By Cauchy Schwarz inequality $\sigma_{1} \sqrt{p q}+\ldots+\sigma_{k} \sqrt{p q}+\sigma_{k+1} \sqrt{p q}+\ldots+\sigma_{2 k} \sqrt{p q}+\sigma_{2 k+1}(0)+\ldots+\sigma_{n}(0) \leq \sqrt{\left(2 m+\sum_{i=1}^{n}\left(d_{i}(G)\right)^{2}\right) 2 k p q}$

But $\sigma_{1}=\sigma_{2}=\ldots=\sigma_{k}$

$$
\begin{aligned}
& \therefore \sigma_{1} k \sqrt{p q}+\sqrt{p q} \sum_{i=k+1}^{2 k} \sigma_{i} \leq \sqrt{\left(2 m+\sum_{i=1}^{n}\left(d_{i}(G)\right)^{2}\right) 2 k p q} \\
& \sigma_{1} k+\sum_{i=k+1}^{2 k} \sigma_{i} \leq \sqrt{\left(2 m+\sum_{i=1}^{n}\left(d_{i}(G)\right)^{2}\right) 2 k} \\
& \sigma_{1} \leq \frac{1}{k}\left(\sqrt{\left(2 m+\sum_{i=1}^{n}\left(d_{i}(G)\right)^{2}\right) 2 k}-\sum_{i=k=1}^{2 k} \sigma_{i}\right) .
\end{aligned}
$$

Corollary 2.19. If $2 k=n$ then by above theorem

$$
L E(G) \leq \sqrt{\left(2 m+\sum_{i=1}^{n}\left(d_{i}(G)\right)^{2}\right) n} .
$$

Corollary 2.20. If $2 k=(n-1)$ then by above theorem

$$
L E(G) \leq \sqrt{\left(2 m+\sum_{i=1}^{n}\left(d_{i}(G)\right)^{2}\right)(n-1)}+\sigma_{n} .
$$

\section{REFERENCES}

[1] H.S Ramane, H.B walikar, Bounds for the eigenvalues of a graph, Graphs, Combinatorics, algorithms and applications, Narosa publishing House, New Delhi, 2005.

[2] D.Cvetković, I.Gutman (eds.),Applications of Graph Spectra, Mathematical Institution,Belgrade, 2009. 
[3] D. Cvetković, I.Gutman (eds.), Selected Topics on Applications of Graph Spectra, Mathematical Institute Belgrade, 2011.

[4] A. Yu, M. Lu, F. Tian, On spectral radius of graphs, Linear algebra and its applications, $\mathbf{3 8 7}(2004), 41-49$.

[5] A.Graovac, I.Gutman, N.Trinajstić, Topological Approach to the Chemistry of Conjugated Molecules Springer, Berlin, 1977.

[6] I.Gutman, The energy of a graph.Ber. Math-Statist. Sekt. Forschungsz.Graz, 103(1978), 1-22.

[7] T. AleksioLc, Upper bounds for Laplacian energy of graphs, MATCH Commun. Math. Comput. Chem, 60(2008), 435-439.

[8] I.Gutman, in The energy of a graph: Old and New Results, ed.by A. Betten, A. Kohnert,R. Laue, A. Wassermann. Algebraic Combinatorics and Applications,Springer, Berlin, (2001), $196-211$.

[9] I.Gutman, O.E. Polansky, Mathematical Concepts in Organic Chemistry,Springer, Berlin, 1986.

[10] Huiqing Liu,Mei Lu and Feng Tian, Some upper bounds for the energy of graphs, Journal of Mathematical Chemistry, 41(2007), No.1.

[11] B. Zhou and I. Gutman, On Laplacian energy of graphs, MATCH Commun. Math. Comput. Chem., 57(2007), 211-220.

[12] B. Zhou, I. Gutman and T. Aleksi, A note on Laplacian energy of graphs, MATCH Commun. Math. Comput. Chem., 60(2008), 441-446.

[13] B. Zhou, New upper bounds for Laplacian energy, MATCH Commun. Math. Comput. Chem., 62(2009), 553-560. 